\title{
Pulmonary tuberculosis in a recent immigrant
}

Dominic Wang, Kara Marie Ruicci

\section{CASE SUMMARY}

An elderly patient with a history significant for recent immigration presented with acute onset hemoptysis and a focal infiltrate on chest x-ray. A broad differential for acute hemoptysis was considered including infectious, inflammatory, and neoplastic etiologies. Sputum samples revealed the presence of drug-susceptible $M$. tuberculosis and treatment guidelines were reviewed. The patient demonstrated response to first-line therapy, rifampin and isoniazid, and was subsequently discharged with follow-up chest x-ray. Tuberculosis is further discussed in the broader contexts of current Canadian screening guidelines, emerging drug-resistant strains, and the initiatives of the World Health Organization (WHO) to reduce its global burden.

\section{CASE PRESENTATION}

A 68-year-old male presented to the emergency department with hemoptysis and mild right-sided pleuritic chest pain. He had a 1-month history of fatigue and cough. He first noticed blood with his cough 10 days ago. He also began to experience night sweats and a fever over the last 3 days. His wife informed the health care team that he had a 3-week-long episode of gradual, long-lasting, low-grade fevers several years ago. He immigrated to Canada from India 14 months ago, and had also traveled extensively throughout Europe and South America over the past 5 years. He had no significant history of sick contacts, was a nonsmoker, and had an average of 4-6 drinks per week. He was taking statins.

He was admitted to the wards in airborne infection isolation. On admission, his temperature was $39.4^{\circ} \mathrm{C}$; blood pressure was 110/60 mmHg; heart rate was 108 beats per minute (regular, no murmurs or additional sounds); respiratory rate was 26 breaths per minute; and $\mathrm{O}_{2}$ saturation was $97 \%$ on room air. On physical examination, amphoric breath sounds were noted in the right upper lung field. No significant abnormalities were noted in the complete blood count or serum electrolytes. A chest X-ray revealed focal infiltration in the apical-posterior segments of the right upper lobe.

\section{CLINICAL DIFFERENTIAL AND DIAGNOSIS}

This patient's history and presentation strongly suggested reactivation tuberculosis (also known as chronic tuberculosis). Pulmonary tuberculosis (TB) is an infection of the lungs with $M$. tuberculosis after inhalation of the bacterium via aerosol droplets. Of those infected, $5 \%$ develop primary TB disease, while the remaining 95\% develop a latent TB infection. Latent TB reactivates in 5\% of all infected individuals. The classic presentation of reactivation TB consists of a cough persisting for more than 3 weeks, along with advanced signs and symptoms, including hemoptysis, anorexia, weight loss, and/or chest pain. ${ }^{1}$ Several case series of hospitalized patients documented cough, weight loss, and fatigue developing in $50-67 \%$ of patients, while chest pain, dyspnea, and hemoptysis presented in $25-33 \%{ }^{2,3}$ Additionally, fevers and night sweats, or night sweats alone developed in approximately $50 \%$ of patients. ${ }^{2,3}$ Most immunocompetent patients have abnormalities on chest x-ray, with $80-90 \%$ demonstrating abnormalities in the apical-posterior segments of the upper lobes. ${ }^{3,4}$ Radiographic findings of reactivation TB without upper lobe infiltrates is considered "atypical." 4,5 Atypical findings such as mediastinal lymphadenopathy are more common in immunocompromised patients. ${ }^{6}$

In contrast to reactivation $\mathrm{TB}$, the presentation of primary $\mathrm{TB}$ is predominated by gradual, low-grade fevers lasting 14-21 days. Some patients develop additional symptoms including pleuritic, retrosternal, or interscapular pain. Fatigue, cough, arthralgia, and pharyngitis rarely develop. Chest x-ray is often normal. ${ }^{7}$ The most common finding is hilar adenopathy (approximately 66\%), and only $13 \%$ show upper lobe infiltrates.

Other differential diagnoses of hemoptysis in developed countries include bronchitis, bronchogenic carcinoma, and bronchiectasis. ${ }^{89}$ TB as well as Paragonimus westermani should also be considered if there is significant travel history in regions where these infectious agents are endemic. TB is considered endemic throughout the world. However, most cases of active TB are reported in South-East Asia and the Western Pacific region (62\%) or Africa (25\%). ${ }^{10}$ Moreover, the WHO estimates that $23 \%$ of the global population ( 1.7 billion people) are infected with latent TB. ${ }^{10} P$. westermani infections are most endemic in Asia. ${ }^{11}$ Fungi endemic to North America that should also be considered include Histoplasma, Coccidioides, and Blastomyces; the latter is found in areas bordering the Great Lakes and St. Lawrence River. ${ }^{12-14}$

\section{TB DISEASE BURDEN AND SCREENING}

TB is one of the top 10 causes of death worldwide, with 10 million new cases and 1.3 million deaths in 2017 alone. New cases of TB are largely concentrated in lower-income countries. Incidence rates in the 30 countries with the greatest TB burden range from 150 to 500 per 100 000. In comparison, the incidence of TB in Canada is 4.8 per 100000 (with a total of 1737 cases). Foreign-born Canadians and visitors account for $70 \%$ of these cases. ${ }^{15}$

Screening immigrants and refugees for $\mathrm{TB}$ is critical for detection and prevention of its spread. The tuberculin skin test (TST) is used for screening. The TST consists of an intradermal injection of tuberculin material. Tuberculin is a mycobacterialspecific antigen. T lymphocytes sensitized by previous TB infection react to the material and produce a visible induration within 4872 hours. The Canadian Collaboration for Immigrant and Refugee Health's "Evidence-based clinical guidelines for immigrants and refugees" recommends TST for all children and adolescents under 20 years of age from countries with a high incidence of TB, as well 
as for all adult immigrants who have risk factors that contribute to a significantly increased risk of active tuberculosis, such as human immunodeficiency virus infection, organ transplantation, kidney dialysis, silicosis, and treatment with tumour necrosis factor-alpha blockers. ${ }^{16}$ All adult refugees between 20-50 years of age should also undergo TST. ${ }^{17}$ However, TST is considered to be unreliable in elderly patients, with many studies finding a significant number of false negatives as well as converters (ie previously negative TST, subsequent TSTs becoming positive). ${ }^{18}$ Another test that may be used for screening are interferon-gamma release assays (IGRA) which are more specific and sensitive. ${ }^{19}$ However, IGRA is currently not recommended by Canadian guidelines, likely due to cost. ${ }^{20}$

\section{CASE CONTINUATION}

Sputum samples were collected from the patient for Nucleic Acid Amplification Tests (NAAT), smear, and culture. NAAT was positive for $M$. tuberculosis complex, indicative of active TB. Quadruple drug therapy consisting of rifampin (RMP), isoniazid (INH), pyrazinamide (PZA), and ethambutol (EMB) was initiated immediately. The smear returned positive for acid-fast bacilli. Twelve days later, the culture confirmed the presence of drugsusceptible $M$. tuberculosis. EMB was discontinued.

\section{WORK-UP OF DRUG-SUSCEPTIBLE TB}

NAAT enables rapid diagnosis of suspected TB. These assays amplify DNA from sputum samples, allowing for identification of the organism and detection of drug resistance to some of the first line therapies. ${ }^{1}$ Confirmation of findings should be made with acidfast staining on smear and culture. The presence of acid-fast bacteria is almost synonymous with mycobacteria, with the exception of Nocardia. However, Nocardia infections are typically associated with an immunocompromised state. ${ }^{21}$ Conventional culture remains the gold standard for TB diagnosis and drug susceptibility testing, and should be ordered to confirm results of the NAAT and smear.

Choice of therapy begins after considering whether the strain is likely to be susceptible or resistant to any of the 4 first-line therapies: RMP, INH, PZA, or EMB. The strain may be presumed drug-susceptible if the incidence of drug-resistant TB is low in the area of suspected origin. Though there are no clear guidelines to define a low incidence rate, less than $2.9 \%$ of all new $2017 \mathrm{~TB}$ cases in India were drug-resistant. ${ }^{10}$ If the strain is confirmed to be susceptible to all first-line drugs, EMB is stopped immediately, PZA after 2 months, and INH and RMP after 6 months.

\section{EMERGENCE OF DRUG-RESISTANT TB}

While the presented patient's TB strain was found to be drugsusceptible, the incidence of drug-resistant TB is steadily rising. Drug-resistant TB can be classified as either rifampicin-resistant (RR-TB), multi-drug resistant (MDR-TB; defined as resistant to both rifampicin and isoniazid), or extremely-drug resistant (XDRTB; defined as MDR-TB plus resistant to a second-line agent such as fluoroquinolone and an injectable agent such as amikacin). In 2017, $30 \%$ of new and previously treated TB cases globally were RR-TB. ${ }^{10}$

The WHO recently launched an initiative entitled, "The End TB Strategy.” The strategy has 3 overall goals for 2035: 1) a 90\% reduction of the number of $\mathrm{TB}$ deaths compared with $2015 ; 2$ ) a $90 \%$ reduction of the TB incidence rate compared with 2015; and 3) the elimination of the catastrophic costs due to TB in TBaffected families. These goals are dependent on the development of novel diagnostics and treatments to eliminate both the active and latent forms of TB currently carried by more than 1.7 billion people worldwide. Currently, there are 20 drugs and 12 vaccine candidates in phase I, II or III clinical trials for TB treatment. While in Canada TB is treatable and has a relatively low burden on the overall healthcare system, it is a vast epidemic worldwide and requires urgent attention as the drug-resistant strains continue to expand.

\section{CASE CONCLUSION}

The patient responded to therapy over the course of his admission with gradual resolution of hemoptysis, chest pains, fever, and cough. A subsequent sputum smear returned negative, and the patient was discharged with instruction for continued therapy. The patient was educated regarding the importance of infection control measures and attending follow-up appointments, and the consequences of untreated or incompletely treated TB. Directly observed therapy (DOT) was recommended to the patient and explained to be an effective way to complete TB therapy in as short a time as possible, while avoiding the development of drug resistance. ${ }^{22}$ The patient was amendable to DOT, and follow-up care was coordinated accordingly. Follow-up chest X-ray and sputum cultures in the community were scheduled in 2 months time to confirm response to therapy.

In summary, this 68-year-old patient presented with drugsusceptible reactivation TB and was treated with first-line therapy with follow-up planned in 2 months for confirmation of response to therapy.

MCQ: Which of the following treatment regimens should be initiated for a patient with culture-confirmed, drug-susceptible pulmonary tuberculosis?

A. Rifampin, isoniazid, pyrazinamide, and ethambutol

B. Rifampin, isoniazid, and pyrazinamide

C. Rifampin and isoniazid

D. Rifampin only

Answer: B. Initial empirical treatment with 'quadruple therapy' consists of: rifampin, isoniazid, pyrazinamide, and ethambutol. If he TB strain is confirmed to be drug-susceptible (ie susceptible to all first-line drugs), ethambutol is stopped immediately. If followup confirms response to therapy, pyrazinamide is stopped after 2 months, and isoniazid and rifampin after 6 months. Refer to the discussion above, "Work-Up of Drug-Susceptible TB." 


\section{REFERENCES}

1. Gale-Rowe M, Menzies D, Sutherland J, et al. Highlights of the new 7th edition of the Canadian Tuberculosis Standards. Can Commun Dis Rep. 2014 Mar;40(6):113-6. https://doi.org/10.14745/ccdr.v40i06a04

2. Arango L, Brewin AW, Murray JF. The spectrum of tuberculosis as currently seen in a metropolitan hospital. Am Rev Respir Dis. 1973 Oct;108(4):805-12.

3. MacGregor RR. A year's experience with tuberculosis in a private urban teaching hospital in the postsanatorium era. Am J Med. 1975 Feb;58(2):221-8. https://doi.org/10.1016/0002-9343(75)90573-2

4. Barnes PF, Verdegem TD, Vachon LA, et al. Chest roentgenogram in pulmonary tuberculosis. New data on an old test. Chest. 1988 Aug;94(2):316-20. https://doi.org/10.1378/chest.94.2.316

5. Farman DP, Speir WA, Jr. Initial roentgenographic manifestations of bacteriologically proven Mycobacterium tuberculosis. Typical or atypical? Chest. 1986 Jan;89(1):75-7. https://doi.org/10.1378/ chest.89.1.75

6. Havlir DV, Barnes PF. Tuberculosis in patients with human immunodeficiency virus infection. N Engl J Med. 1999 Feb;340(5):36773. https://doi.org/10.1056/NEJM199902043400507

7. Poulsen A. Some clinical features of tuberculosis. Acta Tuberc Scand. 1957;33(1-2):37-92; concl.

8. Santiago S, Tobias J, Williams AJ. A reappraisal of the causes of hemoptysis. Arch Intern Med. 1991 Dec;151(12):2449-51.

9. Hirshberg B, Biran I, Glazer M, et al. Hemoptysis: etiology, evaluation, and outcome in a tertiary referral hospital. Chest. 1997 Aug;112(2):440-4. https://doi.org/10.1378/chest.112.2.440

10. Global tuberculosis report 2018 [Internet]. Geneva (Sweden): World Health Organization; 2018 [cited 2018 Dec 5]. Available from: http:// apps.who.int/medicinedocs/en/m/abstract/Js23553en/

11. Keiser J, Engels D, Buscher G, et al. Triclabendazole for the treatment of fascioliasis and paragonimiasis. Expert Opin Investig Drugs. 2005 Dec;14(12):1513-26. https://doi.org/10.1517/13543784.14.12.1513

12. Wheat LJ, Slama TG, Eitzen HE, et al. A large urban outbreak of histoplasmosis: clinical features. Ann Intern Med. 1981 Mar;94(3):3317. https://doi.org/10.7326/0003-4819-94-3-331

13. Saccente M, Woods GL. Clinical and laboratory update on blastomycosis. Clin Microbiol Rev. 2010 Apr;23(2):367-81. https://doi. org/10.1128/CMR.00056-09

14. Frieden TR, Jaffe HW, Stephens JW, et al. Increase in reported coccidioidomycosis--United States, 1998-2011. MMWR Morb Mortal Wkly Rep. 2013;62(12):217-21.

15. Gallant V, Vachon J, Siu W. Tuberculosis drug resistance in Canada: 2006-2016. Can Commun Dis Rep. 2017 Nov;43(11):236-41. https://doi. org/10.14745/ccdr.v43illa05

16. Ai JW, Ruan QL, Liu QH, et al. Updates on the risk factors for latent tuberculosis reactivation and their managements. Emerg Microbes Infect. 2016 Feb;5:e10. https://doi.org/10.1038/emi.2016.10

17. Pottie K, Greenaway C, Feightner J, et al. Evidence-based clinical guidelines for immigrants and refugees. CMAJ. 2011 Sep;183(12):E824-925. https://doi.org/10.1503/cmaj.090313

18. Stead WW, To T. The significance of the tuberculin skin test in elderly persons. Ann Intern Med. 1987 Dec;107(6):837-42. https://doi. org/10.7326/0003-4819-107-6-837

19. Pai M, Zwerling A, Menzies D. Systematic review: T-cell-based assays for the diagnosis of latent tuberculosis infection: an update. Ann Intern Med. 2008 Aug;149(3):177-84. https://doi.org/10.7326/0003-4819-1493-200808050-00241

20. Asadi L, Heffernan C, Menzies D, et al. Effectiveness of Canada's tuberculosis surveillance strategy in identifying immigrants at risk of developing and transmitting tuberculosis: a populationbased retrospective cohort study. Lancet Public health. 2017 Oct;2(10):e450-7. https://doi.org/10.1016/S2468-2667(17)30161-5

21. Wilson JW. Nocardiosis: updates and clinical overview. Mayo Clin Proc. 2012 Apr;87(4):403-7. https://doi.org/10.1016/j. mayocp.2011.11.016

22. Zhang H, Ehiri J, Yang H, et al. Impact of community-based DOT on tuberculosis treatment outcomes: a systematic review and metaAnalysis. PloS One. 2016 Feb;11(2):e0147744. https://doi.org/10.1371/ journal.pone.0147744 